\title{
ON THE UNSTEADY FLOWS GENERATED BY IMPULSIVE MOTION OF A BOUNDARY
}

\author{
M. Emin Erdogan, C. Erdem Imrak \\ Faculty of Mechanical Engineering, Istanbul Technical University, 34439 \\ Gumussuyu, Istanbul, Turkey \\ imrak@itu.edu.tr
}

\begin{abstract}
Some properties of unsteady unidirectional flows of viscous fluids are examined for flows impulsively started from rest by the motion of a boundary or two boundaries. Three illustrative examples are given. The first example is the unsteady Couette flow, the second is the unsteady flow between two parallel plates suddenly set in motion with the same speed and the third is the unsteady flow in a circular cylinder moving parallel to its length. It is shown that if the conditions for which the values of the quantities such as velocity, flux and skin friction obtained for large times are nearly the same with those obtained for small times are established, the expressions of the quantities obtained for one of large times or small times can also be used for the other.
\end{abstract}

Key Words- Unsteady flow, Couette flow,- flow between two plates, flow in circular cylinder

\section{INTRODUCTION}

Three exact solutions of the time-dependent Navier-Stokes equations are discussed to obtain some general results. It is well known that most of the solutions for unsteady flows of viscous fluids are in the form of series. These series may be rapidly convergent for large times but slowly convergent for small times or vice versa. In this paper, it is shown that if the conditions for which the values of the quantities such as velocity, flux and skin friction obtained for large times are nearly the same with those obtained for small times are established, expressions obtained for large times can also be used for small times and the opposite can also be true. This is very important, because, sometimes it can be difficult to obtain the solution for small times but it can be easy to obtain it for large times, then, the solution obtained for large times can also be used for small times, the opposite can also be true. In order to illustrate this fact, three examples which are the exact solutions of the Navier-Stokes equations are given. The exact solutions of the Navier-Stokes equations are very important for many reasons. They have been collected by many authors [1], [2,3].

In this paper, the flows considered are: the unsteady Couette flow, the unsteady flow between two parallel plates suddenly set in motion with the same speed and the unsteady flow in a circular cylinder moving parallel to its length. The series solution of velocity for the unsteady Couette flow obtained for large times is rapidly convergent for large times but slowly convergent for small times. However, it can be used for small times. For example, for $v t / h^{2}=0.001$ to obtain the value of $u / U$ accurate to fourteen decimal places, the required number of terms is about 54 . 
Furthermore; the series solution of velocity for the unsteady Couette flow obtained for small times is rapidly convergent for small times but slowly convergent for large times, however, it can be used for large times.

Indeed, for $v t / h^{2}=10$ in order to obtain the value of $u / U$ accurate to fourteen decimal places, the required number of terms is about 17 . These examples show that if the expressions of the quantities such as velocity, flux and skin friction can be found for one of large times or small times, these expressions can also be used for the other.

The series solution of velocity for the unsteady flow between two parallel plates suddenly set in motion with the same speed obtained for small times is rapidly convergent for small times but slowly convergent for large times. However, it can be used for large times. For example, for $v t / b^{2}=0.01$ and $y / b=0$ in order to obtain the value of $u / U$ accurate to six decimal places, the required number of terms is about 100. This can be established without difficulty.

The unsteady flow in cylindrical regions has been investigated by many authors. The unsteady flow in a circular pipe was investigated by Szymanski [4]. The unsteady flow outside a cylinder moving parallel to its length was investigated by Batchelor [5]. The unsteady flow between two cylinders was examined by Muiller [6]. The unsteady flow around a cylinder was investigated by Nanda [7]. In this paper, the unsteady flow in a circular cylinder moving parallel to its length is examined. The series solution of velocity for this flow obtained for large times can also be used for small times. Indeed, for $\tau=0.1$ and $r / a=0.5, w / W$ gives about 0.3896 accurate to four decimal places which can be compared with 0.3890 obtained for small times.

The examples given show that if the expressions of the quantities such as velocity, flux and skin friction can be found for one of large times or small times, these expressions can also be used for the other.

\section{UNSTEADY COUETTE FLOW}

When two parallel plates are in relative motion, a simple shearing motion with linear profile is obtained and this flow is called as the Couette flow. In practice, one plate is moved and the other is held stationary. It is assumed that the fluid is bounded by two parallel plates at $y=0$ and $y=h$, and the fluid is initially at rest. Then the fluid sets in motion by the velocity of the upper plate in its own plane, the lower plate being held stationary. The governing equation and the boundary and the initial conditions are

$$
\begin{array}{ll}
\frac{\partial u}{\partial t}=v \frac{\partial^{2} u}{\partial y^{2}}, & \\
u(0, t)=0 & \text { for all } t, \\
u(h, t)=U & \text { for } t>0, \\
u(y, 0)=0 & \text { for } 0 \leq y \leq h
\end{array}
$$

where $h$ is the distance between two parallel plates and $U$ is the velocity of the upper plate. The solution of equation (1) subject to boundary and initial conditions, equation (2), is obtained by a method which can be applied to all unsteady unidirectional flows. Since the velocity distribution in the case of the steady state is known, then, the velocity can be written as 


$$
\frac{u}{U}=\frac{y}{h}+\frac{2}{\pi} \sum_{n=1} \frac{(-1)^{n}}{n} e^{-n^{2} \pi^{2} v t / h^{2}} \sin \frac{n \pi y}{h} .
$$

If the lower plate moves and the upper one are held stationary, then, the velocity distribution becomes

$$
\frac{u}{U}=1-\frac{y}{h}-\frac{2}{\pi} \sum_{n=1} \frac{1}{n} e^{-n^{2} \pi^{2} v t / h^{2}} \sin \frac{n \pi y}{h} .
$$

The rapidity with which terms of this series expansion tend to zero increases with $\mathrm{n}$. The first term $n=1$ survives longest and therefore this first term dominates the series.

Although equation (3) is obtained for large times and it is slowly convergent for small times, it can also be used for small times, if the number of terms which are considered is sufficient. This can be realized without any difficulty.

The volume flux across a plane normal to the flow and per unit width of this plane can be written as

$$
\frac{Q}{U h / 2}=1-\frac{8}{\pi^{2}} \sum_{n=0} \frac{1}{(2 n+1)^{2}} e^{-(2 n+1)^{2} \pi^{2} \tau}
$$

where $\tau=v t / h^{2}$. The series in equation (4) is valid for all $\tau$. For $\tau=0$, since

$$
\sum_{n=0} \frac{1}{(2 n+1)^{2}}=\frac{\pi^{2}}{8}
$$

$Q$ becomes zero. The required time for $Q$ to attain the asymptotic value is short and it is about $\tau=1$. The factional force per unit area exerted by the fluid on the moving plate at $y=h$ is

$$
\tau_{h}=\mu\left(\frac{u}{y}\right)_{y=h}=\frac{\mu U}{h}\left[1+2 \sum e_{n=1}^{-n^{2} \pi^{2} \tau}\right]
$$

For small values of time, the flow near the moving plate has a character similar to that of the impulsive motion of a plane wall. The required time to attain the asymptotic value of the skin friction is about $\tau=1$.

The expressions given by equations (3), (4) and (5) obtained for large times can also be used for small times. Indeed, in equation (3), for $v t / h^{2}=0.001$ to obtain the value of $U / h$ to fourteen decimal places, the required number of terms is about 54 . This can be realized without difficulty. This shows that the expressions of the quantities such as velocity, flux and skin friction obtained for large times can also be used to find their values for small times.

\subsection{Solutions for small times}

The series solutions of the velocity, flux and skin friction for large times are slowly convergent for small times. Therefore, to obtain the accurate values of these quantities for small times, using the series expansion obtained for large times sufficient number of terms must be calculated. In order to find the number of terms to be calculated the Laplace transform method is used. The Laplace transform of $u(y, t)$ is defined as

$$
\bar{u}=\int_{0}^{\infty} u e^{-s t} d t
$$


Then, equations (1) and (2) become

$$
\bar{u}^{\prime \prime}-\frac{s}{v} \bar{u}=0, \quad \bar{u}(0)=0, \quad \bar{u}(h)=U / s
$$

where primes denote differentiation with respect to $y$. The solution subject to boundary conditions is

$$
\bar{u} / U=\sinh q y / \sinh q h
$$

where $q=(s / v)^{1 / 2}$. In order to obtain the expressions for small times the method given in $[8]$ is used. Since small $t$ corresponds to large $s$, then, $\bar{u}$ can be written as

$$
\frac{\bar{u}}{U}=\sum_{n=0} \frac{1}{s} e^{-q[(2 n+1) h-y]}-\sum_{n=0} \frac{1}{s} e^{-q[(2 n+1) h+y]}
$$

The inverse of $\bar{u}$ is

$$
\frac{u}{U}=\sum_{0} \operatorname{erfc} \frac{(2 n+1)-y / h}{2\left(v t / h^{2}\right)^{1 / 2}}-\sum_{0} \operatorname{erfc} \frac{(2 n+1)+y / h}{2\left(v t / h^{2}\right)^{1 / 2}}
$$

It is clearly seen that for $y / h<<1$ and $v t / h^{2}<<1$, equation (7) takes the form

$$
\frac{u}{U}=\operatorname{erfc} \frac{h-y}{2 \sqrt{v t}}
$$

which is the velocity distribution of the flow produced by the impulsive motion of a plate near $y=h$.

The series expansion in equation (7) is rapidly convergent for $v t / h^{2}<<1$, but slowly convergent for $v t / h^{2}>1$. However, it can also be used for large times. For small times, the volume flux $Q$ across a plane normal to the flow and per unit width of this plane is

$$
\begin{aligned}
\frac{Q}{U h / 2} & =4\left(\frac{v t}{h^{2}}\right)^{1 / 2}\left[\sum_{n=0} \operatorname{ierfc} \frac{n+1}{\left(v t / h^{2}\right)^{1 / 2}}+\sum_{n=0} \operatorname{ierfc} \frac{n}{\left(v t / h^{2}\right)^{1 / 2}}\right. \\
& \left.-2 \sum_{n=0} \operatorname{ierfc} \frac{2 n+1}{2\left(v t / h^{2}\right)^{1 / 2}}\right]
\end{aligned}
$$

where

$$
\begin{aligned}
& i^{n} \operatorname{erfc} x=\int_{x}^{\infty} i^{n-1} \operatorname{erfc} \xi d \xi \\
& i^{0} \text { erfc } x=\operatorname{erfc} x
\end{aligned}
$$

are integrals of complementary error function which are obtained from tables [9].

The frictional force $\mu(\partial u / d y)_{y=h}$ per unit area exerted by the fluid on the moving plate can be obtained by equation (6). The Laplace transform of $\tau_{h}=\mu(\partial u / \partial y)_{y=h}$ is given as $\bar{\tau}_{h}=\mu(\partial \bar{u} / \partial y)_{y=h}$ and inserting equation (6), one finds

$$
\bar{\tau}_{h}=\mu\left(\frac{\partial \bar{u}}{\partial y}\right)_{y=h}=\frac{\mu U}{h}\left[\sum_{n=0} \frac{1}{s^{1 / 2}} e^{-2 n q h}+\sum_{n=0} \frac{1}{s^{1 / 2}} e^{-(2 n+2) q h}\right]
$$

The inverse of $\bar{\tau}_{h}$ is 


$$
\tau_{h}=\mu\left(\frac{\partial u}{\partial y}\right)_{y=h}=\frac{\mu U / h}{\left(\pi v t / h^{2}\right)^{1 / 2}}\left[\sum_{n=0} e^{\frac{n^{2}}{v t / h^{2}}}+\sum_{n=0} e^{\frac{(n+1)^{2}}{v t / h^{2}}}\right]
$$

The expression of $\tau_{h}$ obtained for small times can also be used for large times. Indeed, for $v t / h^{2}=1$, one obtains $\tau_{h} /(\mu U / h)=1.000103$ accurate to six decimal places which can be compared with 1.000104 obtained for large times.

\section{FLOW BETWEEN TWO PARALLEL PLATES SUDDENLY SET IN MOTION WITH THE SAME SPEED}

It is assumed that the fluid is bounded by two parallel plates at $y=-b$ and at $y=b$, and it is initially at rest and the fluid starts suddenly by the motion at the same speed of the upper and the lower plates in their own planes. The governing equation is equation (1), and the boundary and the initial conditions are

$$
\begin{array}{lll}
u( \pm b, t)=U & \text { for } & t>0 \\
u(y, 0)=0 & \text { for } & -b<y<b
\end{array}
$$

where $2 b$ is the distance between two parallel plates. Since the velocity distribution in the case of the steady-state is $U$, then, the velocity $u(y, t)$ can be written as

$$
\frac{u}{U}=1-\frac{4}{\pi} \sum_{n=0} \frac{(-1)^{n}}{2 n+1} e^{-(2 n+1)^{2} \pi^{2} \tau / 4} \cos (2 n+1) \pi \eta / 2
$$

where $\tau=v t / b^{2}$ and $\eta=y / b$. The first term $n=0$ survives longest and therefore this first term dominates the series.

Although equation (9) is obtained for large times, it will be seen later that it can be used for small times, if the number of terms which are considered is sufficient. The variation of $u / U$ with $y / b$ for various values of $v t / b^{2}$ is illustrated in Figure 1. For small values of time, the flow near the plates has the character of the Couette flow and there is no effect due to the plates on the region near the centerline. It can be seen from Fig.1 that the required time for $u(y, t)$ to attain the asymptotic value is about $v t / b^{2}=2$.

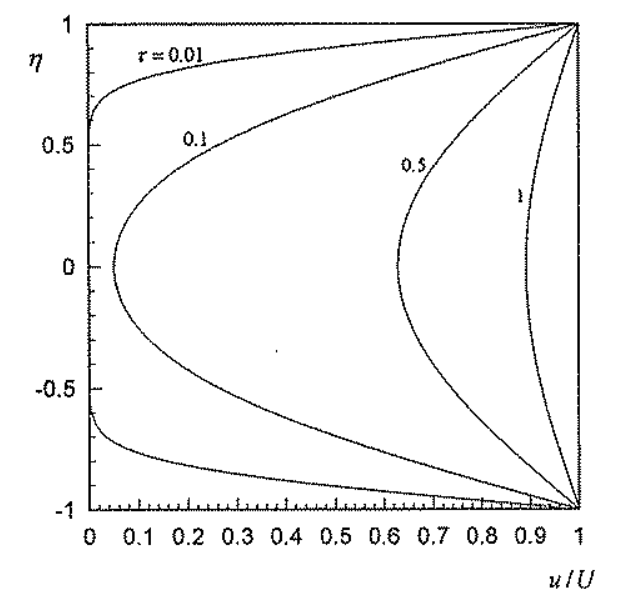

Figure 1. Flow between two parallel plates suddenly set in motion with the same speed. The variation of $u / U$ with $y / b$ for various values of $v t / b^{2}$. 
The volume flux $Q$ across a plane normal to the flow and per unit width of this plane is

$$
\frac{Q}{2 b U}=1-\frac{8}{\pi^{2}} \sum_{0} \frac{1}{(2 n+1)^{2}} e^{-(2 n+1)^{2} \pi^{2} x / 4} .
$$

The series in equation (10) is valid for all $\tau$. For $\tau=0$, since

$$
\sum_{n=0} \frac{1}{(2 n+1)^{2}}=\frac{\pi^{2}}{8}
$$

$Q$ becomes zero. The required time to attain the asymptotic value of $Q$ is about $\tau=2$.

The frictional force per unit area exerted by the fluid on the plate at $y=b$ is

$$
\tau_{b}=\mu\left(\frac{\partial u}{\partial y}\right)_{y=b}=2 \frac{\mu U}{b} \sum_{n=0} \exp \left[-(2 n+1)^{2} \pi^{2} \tau / 4\right]
$$

The required time to attain the asymptotic value of the skin friction is about $\tau=2$.

\subsection{Solutions for small times}

The series solutions of velocity, flux and skin function obtained for large times are slowly convergent for small times. Therefore, to obtain the accurate values of these quantities for small times using the series expansion obtained for large times, sufficient number of terms must be calculated. In order to find the number of terms to be calculated the Laplace transform method is used. The Laplace transform of $u$ is $\bar{u}$, then equation (1) and boundary conditions take the following forms

$$
\begin{aligned}
& \bar{u}-\frac{s}{v} \bar{u}=0, \\
& \bar{u}( \pm b)=\frac{U}{s}
\end{aligned}
$$

where primes denote differentiation with respect to $y$. The solution subject to boundary conditions is

$$
\frac{\bar{u}}{U}=\frac{\cosh q y}{\cosh q b}
$$

where $q=(s / v)^{1 / 2}$. For small times the method given in [8] is used. Since small $t$ corresponding to large $s$, then, $\bar{u}$ can be written as

$$
\frac{\bar{u}}{U}=\sum_{n=0}(-1)^{n} \frac{e^{-q[(2 n+1) b-y]}}{s}+\sum_{n=0}(-1)^{n} \frac{e^{-q[(2 n+1) b+y]}}{s}
$$

The inverse of $\bar{u}$ is

$$
\frac{u}{U}=\sum_{n=0}(-1)^{n} \operatorname{erfc} \frac{(2 n+1)-\eta}{2 \sqrt{\tau}}+\sum_{n=0}(-1)^{n} \operatorname{erfc} \frac{(2 n+1)+\eta}{2 \sqrt{\tau}} .
$$

The series expansions in Eq. (13) is slowly convergent for $\tau \gg 1$, however, it can also be used for $\tau \gg 1$. Indeed, for $\tau=0.01$ and $\eta=0$, equation (13) gives $u / U=2$ erfc 5 $=3.074918 \times 10^{-12}$ accurate to six decimal places. This result can also be obtained by equation (9), the number of terms to be calculated in the series in about 100 . This can be realized using a computer.

For small times, the volume flux $Q$ across a plane normal to the flow and per unit 
width of this plane is

$$
\frac{Q}{2 U b}=2 \tau^{1 / 2}\left[\frac{1}{\sqrt{\pi}}+2 \sum_{n=1}(-1)^{n} \text { i erfc } \frac{n}{\sqrt{\tau}}\right]
$$

The expression for $Q$ given in equation (14) obtained for small times, can also be used for large times. Indeed, for $\tau=1$, this gives about 0.9313 accurate to four decimal places which is exactly the same with that obtained for large times.

The frictional force $\mu(\partial u / \partial y)_{y=b}$ per unit area exerted by the fluid on the plate at $y=b$ is

$$
\tau_{b}=\mu\left(\frac{\partial u}{\partial y}\right)_{y=b}=\frac{\mu U / b}{(\pi \tau)^{1 / 2}}\left[1+2 \sum_{i}(-1)^{n} e^{-n_{2} / \tau}\right] .
$$

Although the expression of $\tau_{b}$ is obtained for small times, it can also be used for large times. For $\tau=1$, it gives $\tau_{b} /(\mu U / b)=0.1696$ accurate to four decimal places which is exactly the same with that for large times.

\section{UNSTEADY FLOW IN A CIRCULAR CYLINDER MOVING PARALLEL TO ITS LENGTH}

Suppose that the fluid is in a circular cylinder and it is initially at rest, and the fluid starts suddenly due to motion of the cylinder parallel to its length. Cylindrical polar coordinates are used. The axis of the cylinder is the $z$-axis. The governing equation is

$$
\frac{\partial w}{\partial t}=v\left(\frac{\partial^{2} w}{\partial r^{2}}+\frac{1}{r} \frac{\partial w}{\partial r}\right)
$$

where, $w(r, t)$ is the velocity along the z-axis, $v$ is the kinematic viscosity of the fluid, $r$ is the coordinate and $t$ is the time. The boundary and the initial conditions are

$$
\begin{array}{rlrl}
w(a, t)=W & \text { for } & t>0 \\
w(r, 0) & =0 & \text { for } & 0 \leq r<a
\end{array}
$$

where $W$ is the constant velocity at $r=a$ and $a$ is the radius of the cylinder. The solution of equation (15) subject to boundary and initial conditions (16) may be obtained by the Laplace transform method. However, the solution is obtained by a method which can be applied to all unsteady unidirectional flows. Since the velocity distribution in the case of the steady-state is $W$, namely, the fluid moves eventually at the same speed of the cylinder, then, the velocity $w(r, t)$ can be written as

$$
w(r, t)=W-f(r, t)
$$

where $f(r, t)$ satisfies the following differential equation

$$
\frac{\partial f}{\partial t}=v\left(\frac{\partial^{2} f}{\partial r^{2}}+\frac{1}{r} \frac{\partial f}{\partial r}\right)
$$

and the boundary and the initial conditions are

$$
f(a, t)=0, \quad f(r, 0)=W .
$$

When $t$ goes infinity $f(r, t)$ tends to zero. The first boundary condition suggests a 
solution in the following form

$$
f(r, t)=\sum_{n=1} \exp \left[-\lambda_{n}^{2} v t / a^{2}\right] F_{n}(r)
$$

where $F_{n}(r)$ satisfies the following differential equation

$$
F_{n}^{\prime \prime}+\frac{1}{r} F_{n}^{\prime}+\frac{\lambda_{n}^{2}}{a^{2}} F_{n}=0
$$

where primes denote differentiation with respect to $r$. The solution of this differential equation is

$$
F_{n}(r)=A_{n} J_{0}\left(\lambda_{n} r / a\right) .
$$

Since $F_{n}=0$ at $r=a$, one finds

$$
J_{0}\left(\lambda_{n}\right)=0
$$

where $\lambda_{n}$ are zeros for which the values are obtained from tables [9]. Thus the velocity distribution is given by

$$
\frac{w}{W}=1-2 \sum_{n=1} \frac{J_{0}\left(\lambda_{n} \xi\right)}{\lambda_{n} J_{1}\left(\lambda_{n}\right)} e^{-\lambda_{n}^{2} \tau}
$$

where $\tau=v t / a^{2}$ and $\xi=r / a$, and $J_{1}$ is the Bessel function of the first kind of order one. The first term $n=1$ survives longest and therefore this first term dominates the series. Equation (17) is obtained for large times, however, it can be used for small times, if the number of terms which are considered is sufficient. The variation of $w / W$ with $r / a$ for various values of $\tau$ can be illustrated in a figure similar to that of Figure 1. The effect of the friction of the flow in the cylinder is greater than that of the flow between two parallel plates. Thus the required time to attain the asymptotic value for the flow in the cylinder is shorter than that of the two-dimensional case. Indeed, the required time to attain the asymptotic value is about $\tau=1$.

The volume flux $Q$ across a plane normal to the flow is

$$
\frac{Q}{W\left(\pi a^{2}\right)}=1-4 \sum_{n=1} \frac{1}{\lambda_{n}^{2}} \exp \left(-\lambda_{n}^{2} \tau\right)
$$

where $\tau=v t / a^{2}$. For $\tau=0$, since

$$
\sum_{n=1} \frac{1}{\lambda_{n}^{2}}=\frac{1}{4}
$$

$Q$ becomes zero. The required time to attain the asymptotic value of $Q$ is short and it is about $\tau=1$.

The frictional force per unit area exerted by the fluid on the surface of the cylinder at $r=a$ is

$$
\tau_{a}=\mu(\partial w / \partial r)_{r=a}=2 \frac{\mu W}{a} \sum_{n=1} \exp \left(-\lambda_{n}^{2} \tau\right) .
$$

The required time to attain the asymptotic value of the skin friction is about $\tau=1$.

\subsection{Solutions for small times}

For small times the Laplace transform method is very useful [8]. If the Laplace transform of $w$ is $\bar{w}$, then, equations (15) and (16) take the following forms 


$$
\bar{w}^{\prime \prime}+\frac{1}{r} \bar{w}^{\prime}-q^{2} w=0, \quad \bar{w}(a)=W / s
$$

where $q=(s / v)^{1 / 2}, s$ is the transform parameter and primes denote differentiation with respect to $r$. The solution subject to boundary condition is

$$
\bar{W} / W=I_{0}(q r) / s I_{0}(q a)
$$

where $I_{o}(x)$ is the modified Bessel function of the first kind of order zero and the condition that the velocity is finite at $r=a$ is used. For small times, $\bar{w}$ can be written as

$$
\frac{\bar{w}}{W}=\left(\frac{a}{r}\right)^{1 / 2} \frac{e^{-q(a-r)}}{s}\left(1-\frac{a-r}{8 q a r}+\frac{9 a^{2}-2 a r-7 r^{2}}{128 q^{2} a^{2} r^{2}}+\cdots\right) \text {. }
$$

The inverse of $\bar{w}$ can be found in the following form

$$
\frac{w}{W}=\frac{1}{\xi^{1 / 2}} \operatorname{erfc} \frac{1-\xi}{2 \sqrt{\tau}}+\frac{(1-\xi) \tau^{1 / 2}}{4 \xi^{1 / 2}} \text { ierfc } \frac{1-\xi}{2 \sqrt{\tau}}+\frac{\left(9-2 \xi-7 \xi^{2}\right) \tau}{32 \tau^{3 / 2}} \operatorname{ierfc} \frac{1-\xi}{2 \sqrt{\tau}}+\cdots
$$

where $\tau=v t / a^{2}$ and $\xi=r / a$. The series expansion in equation (21) is rapidly convergent for $\tau<<1$, but slowly convergent for $\tau>>1$. However, it can be shown that it can also be used for $\tau \gg 1$. Indeed, for $\tau=0.1$ and $\xi=0.5$, equation (21) for $w / W$ gives about 0.3880 accurate to four decimal places which can be compared with 0.3896 obtained for large times.

For small times, the volume flux $Q$ across a plane normal to the flow is

$$
\frac{Q}{W\left(\pi a^{2}\right)}=\frac{4}{\pi^{1 / 2}} \tau^{1 / 2}-\tau-\frac{1}{\pi^{1 / 2}} \tau^{3 / 2}-\frac{1}{8} \tau^{2}-\cdots
$$

where $\tau=v t / a^{2}$. For $\tau=0.01$, equation (22) gives for $Q / W\left(\pi a^{2}\right)$ about 0.2151 accurate to four decimal places which can be compared with 2.000 obtained for large times.

The frictional force $\mu(\partial w / \partial r)_{r=a}$ per unit area exerted by the fluid on the surface $r=a$ is

$$
\tau_{a}=\mu\left(\frac{\partial w}{\partial r}\right)_{r=a}=\frac{\mu W}{a}\left(\frac{1}{(\pi \tau)^{1 / 2}}-\frac{1}{2}-\frac{\tau^{1 / 2}}{4 \pi^{1 / 2}}-\frac{\tau}{8}-\cdots\right)
$$

This expression for the skin friction is obtained for small times, however, it can also be used for large times. Indeed, for $v t / b^{2}=0.01, \tau_{a} /(\mu W) / a=5.1265$ accurate to four decimal places, which can be compared with 5.1266 obtained for large times. Since the series expansion for small times can be replaced with the expression for large times, the series expansion for large times can be also be used for small times and the opposite is also true.

\section{CONCLUSIONS}

The unsteady Couette flow, the unsteady flow between parallel plates suddenly set in motion with the same speed and the unsteady flow in a circular cylinder moving parallel to its length are examined. These flows have a common property that their generation is the same type. They start suddenly by the impulsive motion of a boundary or two boundaries. For these flow, the expressions of the quantities such as velocity, 
flux and skin friction are in the form of series in terms of time. These series may be rapidly convergent for large times but slowly convergent for small times. If necessary conditions are established, the series which is slowly convergent for small times can be used for small times or vice versa. This is very important, because, sometimes it can be difficult to obtain the solution for small times but it can be easy to obtain it for large times, then, the solution obtained for large times can be also used for small times. The opposite can also be true. To elucidate the subject three examples are given.

The series solution of velocity for the unsteady Couette flow obtained for large times is rapidly convergent for large times but slowly convergent for small times. However, it can also be used for small times. For examples, for $v t / h^{2}=0.001$ to obtain the value of $u / U$ accurate to fourteen decimal places, the required number of terms is about 54. Although the series solution of velocity for the unsteady Couette flow obtained for small times is rapidly convergent for small times but slowly convergent for large times, it can also be used for large times. Indeed, for $v t / h^{2}=10$, in order to obtain the value of $u / U$ to fourteen decimal places, the required number of terms in the series is about 17.

These examples show that if the expressions of the quantities such as velocity, flux and skin friction can be found for one of large times or small times, these expressions can also be used for the other.

\section{REFERENCES}

1. R. Berker, Intégration des équations du movement d'un fluide visqueux incompressible, Handbuch der Physik, VII/2, Springer-Verlag, Berlin, 1963.

2. C. Y. Wang, Exact solutions of the unsteady Navier-Stokes equations, Appl. Mech. Rev. 42, 270-282, 1969.

3. C. Y. Wang, Exact solutions of the steady-state Navier-Stokes equations, Annu. Rev. Fluid Mech. 42, $159-177,1991$.

4. P. Szymanski, Quelques solutions exactes des équations de l'hydrodynmaique de fluide visqueux dans le cas d'un tube cylindirique, $J$. de math. Pures et appliqués, Séries 9, 11, 67 $107,1932$.

5. G. K. Batchelor, The skin friction on infinite cylinders moving parallel to their length, Quart. J. Mech. And Appl. Math. 7, 179 - 192, 1954.

6. W. Müller, Zum Problem der Anlaufströmung einer Flüssigkeit im geraden Rohr mit Kreisring-und Kreisquerschnitt, Z.angew. Math. Mech (ZAMM), 16, 227 - 238, 1936.

7. R. S. Nanda, Unsteady circulatory flow about a circular cylinder with suction, Appl. Scien. Res. 9A, $85-92,1960$.

8. H. S. Carslaw and J. C. Jaeger, Conduction of Heat in Solids, Oxford University Press, 1973.

9. M. Abramovitz and I. Stegun, Handbook of Mathematical Functions, Dover, 1965. 